\title{
Morphologie et comportement locomoteur : synthèse de l'analyse intégrative d'un modèle primate non humain actuel
}

\author{
Morphology and Locomotor Behaviour: Synthesis of an Integrative Analysis of a Non-Human \\ Primate Model
}

\author{
F. Druelle \\ Reçu le 12 mars 2018; accepté le 11 juin 2018 \\ (C) Société d'Anthropologie de Paris et Lavoisier SAS 2018
}

Résumé De nombreux désaccords existent quant à l'interprétation des aptitudes locomotrices des premiers homininés. En effet, la signification fonctionnelle à donner aux caractères à la fois dérivés et archaïques chez une même espèce fossile, comme par exemple chez l'Australopithecus afarensis, reste difficile. Les travaux intégratifs portant sur les primates non humains actuels (morphologie, biomécanique, répertoire posturo-locomoteur) sont des approches pertinentes à ces questions paléoanthropologiques, car les primates adoptent une multitude de modes locomoteurs avec des spécialisations plus ou moins importantes. Dans ce contexte, le babouin olive, Papio anubis, en tant que catarrhinien quadrupède spécialisé, mais possédant pourtant un répertoire posturolocomoteur varié, représente un bon modèle d'étude. Cette espèce est accessible à la station de primatologie du CNRS (UPS 846) de Rousset pour des études à la fois transversales et longitudinales. Des échantillons ontogénétiques permettent d'observer des variations morphologiques liées au développement allométrique et ainsi d'éclaircir la nature des relations entre morphologie, biomécanique de la marche et répertoire posturo-locomoteur. Cette note est une synthèse des résultats obtenus dans le cadre d'une étude intégrative et longitudinale. Au-delà de la spécialisation à la quadrupédie, il existe chez le babouin olive, comme chez l'ensemble des primates, des compromis morphologiques permettant la réalisation d'un répertoire varié, incluant des proportions quantifiables de bipédie, de grimper, de suspension, etc. Dans une perspective paléoanthropologique, ce constat sur des espèces actuelles suggère que les primates éteints, incluant les premiers homininés, devaient posséder de multiples capacités posturolocomotrices avec différents degrés de spécialisation. Dans ce contexte, la compréhension des relations fonctionnelles

\section{F. Druelle $(\bowtie)$}

Laboratory for Functional Morphology, University of Antwerp Campus Drie Eiken, 1, Universiteitsplein, 2610 Wilrijk, Belgium e-mail : francois.druelle@uantwerpen.be entre les morphologies actuelles, à la fois au niveau des morphotypes globaux (propriétés d'inerties) et de la morphologie osseuse, avec les répertoires posturo-locomoteurs est fondamentale. En effet, plutôt que rechercher un mode locomoteur unique chez nos ancêtres homininés, ces études sur les primates non humains permettront l'interprétation de répertoires posturo-locomoteurs ancestraux.

Mots clés Morphotype - Répertoire posturo-locomoteur · Bipédie · Quadrupédie · Évolution · Ontogenèse · Modèle primate

Abstract There is much disagreement over interpretations of the locomotor capacities of the first hominins. It is indeed always difficult to interpret the functional significance of derived and archaic characters in a fossil of an extinct species, for example, Australopithecus afarensis. Integrative studies (morphology, biomechanics, posturo-locomotor repertoire) of extant non-human primates are highly relevant approaches to these paleoanthropological questions because primates are capable of multiple locomotor modes that include locomotor specializations. In this context, the olive baboon, Papio anubis, as a specialized quadrupedal Catarrhini species that nevertheless exhibits a varied postural and locomotor repertoire, is a good model. This species is available at the CNRS Primatology Station (CNRS UPS 846) for both cross-sectional and longitudinal studies. Ontogenetic samples, because of allometric growth, allow observations of natural morphological variations that can clarify the nature of the relationships between morphology, biomechanics and posturo-locomotor repertoires. This article provides a summary of the results obtained from an integrative and longitudinal study. Over and above their quadrupedal specialization, baboons, like other primates, exhibit morphological compromises that allow them to perform a postural and locomotor repertoire that includes bipedalism, climbing, suspension, etc. in varied proportions. From a paleoanthropological 
perspective, such observations in extant species suggest that extinct primates, including early hominins, would have exhibited multiple postural and locomotor capacities with varying degrees of specialization. An understanding of the functional relationships between morphologies, in terms of both overall morphotypes (inertial properties) and osteology, and posturo-locomotor repertoires in extant non-human primates is therefore fundamental in this regard. Rather than attempting to identify a single locomotor mode in hominin species, these studies on extant non-human primate models can support interpretations of archaic postural and locomotor repertoires.

Keywords Morphotype · Posturo-locomotor repertoire · Bipedalism · Quadrupedalism · Evolution · Ontogeny · Primate model

\section{Introduction}

Les primates non humains actuels possèdent des répertoires posturo-locomoteurs, aussi appelés répertoires positionnels [1]. Ces répertoires se caractérisent par de multiples modes locomoteurs qui sont possibles grâce à une morphologie appropriée. En effet, le corps est composé d'un ensemble de segments articulés possédant des propriétés intrinsèques, neuromusculaires et mécaniques, et peut donc, en tant que support du mouvement, faciliter et/ou contraindre les performances locomotrices telles que la suspension, le grimper, la marche et la course quadrupèdes, la bipédie, etc. Dans ce contexte, une bonne connaissance des propriétés d'inertie $\mathrm{du}$ corps, telles que les masses segmentaires, la position du centre de masse, ou le rayon de giration des membres, peut permettre des interprétations fonctionnelles (mécaniques) et adaptatives des morphotypes et des multiples capacités locomotrices de ces espèces.

La diversité de ces morphotypes et des répertoires posturo-locomoteurs chez les primates actuels est considérable. Comprendre cette diversité, ainsi que l'interaction entre les propriétés d'inertie du corps et les capacités de mouvement, est fondamental pour comprendre la diversité des répertoires des espèces éteintes et notamment des homininés fossiles. À long terme, cette connaissance permettra notamment la reconstruction de scénarios évolutifs intégrant les transitions locomotrices, depuis un répertoire positionnel à l'autre [2]. Au service de cette perspective, l'étude d'un échantillon ontogénétique longitudinal d'une espèce de primate est susceptible d'appréhender des variations morphologiques naturelles permettant d'éclaircir la nature des relations fonctionnelles entre morphologie et comportement locomoteur [3]. En effet, les dimensions linéaires du corps et les paramètres d'inertie changent de manière considérable au cours de l'ontogenèse des primates (humains et non humains). Dans ce contexte, le babouin olive, Papio anubis, est un excellent modèle d'étude, car, bien que quadrupède spécialisé, il possède des capacités posturo-locomotrices diverses. En effet, la diversité des environnements dans lesquels ce primate vit requiert des capacités locomotrices particulières, telles que la marche endurante, la course, le grimper sur falaise, etc. De plus, comme chez tous les primates catarrhiniens non humains actuels, son répertoire inclut une bipédie occasionnelle et spontanée [4], dont les paramètres biomécaniques et comportementaux sont susceptibles de varier au cours du développement.

Le premier objectif de ce travail a été l'étude globale de la morphologie du babouin olive au cours de la croissance, de la naissance à l'âge adulte, à travers les modifications des propriétés d'inertie [5]. Celles-ci sont susceptibles de refléter des stratégies mécaniques adaptatives [6] comme, par exemple, la facilitation de certains mouvements par des mécanismes pendulaires ou des stratégies d'économie d'énergie [7]. Le second objectif a été l'évaluation, à un niveau individuel, de la signification fonctionnelle de ces changements morphologiques. Pour cela, il a fallu croiser les données de morphologie avec :

- le répertoire positionnel [8];

- le mode locomoteur préférentiel de l'espèce, c'est-à-dire la quadrupédie [9] ;

- un mode locomoteur occasionnel, la bipédie [10].

La figure 1 résume le matériel et les méthodes utilisés dans le cadre de ces études.

\section{Développement morphologique du babouin}

L'étude ontogénétique et longitudinale menée sur 30 individus a produit la plus importante base de données de morphométrie segmentaire pour une espèce de catarrhinien. Les résultats montrent que les modèles individuels de croissance sont très cohérents et suivent le modèle général de croissance précédemment décrit pour le babouin olive $[11,12]$. Les courbes de croissance sont généralement similaires pour les longueurs et les masses segmentaires, bien que les échelles de temps soient légèrement différentes. Les changements morphométriques les plus importants se produisent au cours des deux premières années de vie et concernent les parties distales du corps. Les femelles et les mâles ont des formes et des tailles similaires à la naissance. Ce sont le taux et la durée de croissance qui produisent des différences considérables (donc principalement liées à la taille) au cours de l'ontogenèse, tandis que les formes corporelles restent très similaires entre les sexes. Les changements morphologiques observés chez le babouin en croissance semblent répondre aux changements des demandes 


\section{Matériel et Méthodes}

\section{Colonie de 60 individus vivant au sein d'un parc en captivité}

\section{Station de Primatologie du CNRS, Rousset, France}

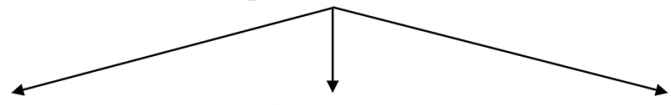

Echantillon longitudinal de 30 individus

(14 femelles et 16 mâles)<smiles>CCC[Te]</smiles>

Analyse développementale :

Prise de masse, distribution

des masses, propriétés

d'inertie, périodes

pendulaires naturelles

Modèles polynomiaux
Echantillon transversal de

22 individus

(11 femelles et 11 mâles)<smiles>[3H][3H]</smiles>

Analyse croisée :

Morphologie $v s$ comportement bipède

(fréquence + durée moyenne)
Echantillon longitudinal de 6 individus

(3 femelles et 3 mâles)

\section{Analyse en composantes principales + tests paramétriques}

Fig. 1 Schéma résumé du matériel et des méthodes utilisés / Summary of the material and methods used

fonctionnelles, notamment lors de l'apparition de la locomotion quadrupède autonome. En effet, sa morphologie reflète initialement de bonnes capacités d'agrippement : les masses sont concentrées de manière distale au sein des membres, et les mains et les pieds représentent des segments relativement massifs. Cependant, cette morphologie change selon des processus allométriques, répondant ainsi graduellement aux nouvelles exigences mécaniques liées à la spécialisation pour la quadrupédie : les masses migrent de manière proximale dans les membres, principalement par une allométrie positive de la masse de la cuisse et du bras ainsi que par une allométrie négative au niveau de la longueur des segments distaux. Par ailleurs, en période infantile, la tête est initialement relativement massive, et le tronc est relativement léger ; cette tendance se modifie avec l'âge. Concernant les propriétés d'inertie des membres antérieurs et postérieurs, une très bonne convergence des périodes pendulaires naturelles est présente dès la naissance chez le babouin olive. Une telle convergence des périodes pendulaires naturelles peut refléter une optimisation biomécanique précoce des membres pour la quadrupédie [13], permettant potentiellement au jeune d'initier précocement, relativement à la maturation du système neuromusculaire, les comportements quadrupèdes en facilitant la coordination entre membres antérieurs et postérieurs. Ces éléments morphologiques fondamentaux fournissent donc une base de données idéale pour questionner les changements comportementaux et biomécaniques qui se produisent parallèlement chez le babouin au cours de son développement.

\section{Morphologie et comportement locomoteur}

\section{Répertoire posturo-locomoteur}

Selon les principes biomécaniques, la morphologie est susceptible d'être liée au répertoire positionnel dans son ensemble. Étant donné que le morphotype des jeunes babouins subit d'importantes modifications durant les deux premières années de vie en raison de processus rapides de croissance, des changements comportementaux sont aussi susceptibles de se produire. Après avoir quantifié les répertoires posturolocomoteurs des babouins semi-autonomes $(\approx 0,5$ an) et autonomes ( $\approx 2$ ans), il apparaît que la proportion de marche quadrupède augmente graduellement durant la période infantile, alors que les comportements d'agrippement, tels que le grimper et la suspension, diminuent dans le répertoire posturo-locomoteur. De plus, ce modèle de changement est fortement corrélé aux changements morphologiques et notamment à la migration proximale des masses au niveau des membres (Fig. 2). Dans un contexte biomécanique, des masses plus distales reflètent d'importants muscles fléchisseurs pour des capacités d'agrippement plus importantes, 


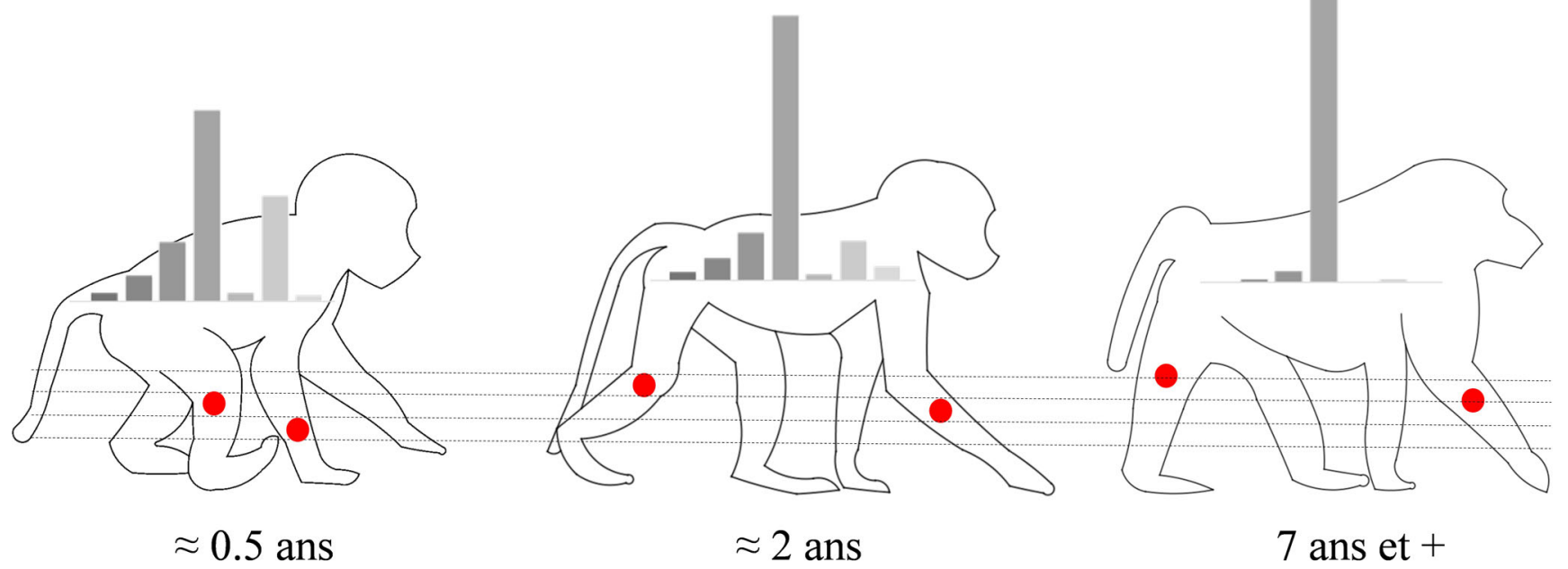

Fig. 2 Relation entre la migration proximale du centre de masse des membres et la diversité du répertoire locomoteur au cours de la croissance du babouin olive. Le point rouge représente la position moyenne du centre de masse du membre considéré. Les diagrammes en barres représentent le répertoire locomoteur moyen pour chaque classe d'âge, de gauche à droite : bipédie, saut, course (quadrupède et tripède), marche (quadrupède et tripède), suspension, grimper, autres (marche accroupie, etc.). Les répertoires des deux classes d'âge à gauche proviennent de Druelle et al. [8] et celui des adultes à droite provient de Druelle et Berillon [14] / Relationship between the proximal migration of the limb centre of mass and the diversity of the locomotor repertoire during growth in olive baboons. The red dot is the average position of the centre of mass for the limb considered. The bar diagrams represent the locomotor repertoire in each age class. From left to right: bipedalism, leaping, quadrupedal and tripedal running, quadrupedal and tripedal walking, suspensory behaviours, climbing, others (squat walking, etc.). The two repertoires on the left are from Druelle et al. [8] and the repertoire for adults is from Druelle et Berillon [14]

alors que posséder des masses plus proximales semble être une adaptation pour réduire la consommation énergétique lors de la marche en réduisant la résistance lors de la phase d'oscillation du membre [15]. Des corrélations fortes ont été trouvées entre le gradient de variation du morphotype (migration proximale des masses dans les membres) et les proportions des modes locomoteurs utilisés (depuis le grimper et la suspension à la marche quadrupède), confirmant ainsi ces hypothèses biomécaniques.

\section{Comportement bipède}

Alors que la biomécanique de la station et de la marche bipède a été largement étudiée chez les primates non humains [16-21], les caractéristiques morphologiques susceptibles de contraindre, ou de faciliter, le contrôle de l'équilibre, et ainsi la spontanéité du comportement bipède, sont peu connues [22]. Dans ce contexte, les changements qui ont lieu au cours de l'ontogenèse des primates non humains peuvent servir d'expérience naturelle (voir ci-dessus) et fournir un aperçu de l'impact de la morphologie sur la bipédie. En effet, les changements du modèle de distribution des masses sont susceptibles d'influencer les variables comportementa- les, c'est-à-dire : la fréquence et la durée des occurrences du comportement bipède en contexte d'activité. Concernant les comportements bipèdes réalisés en statique et assistés par les mains, la durée et la fréquence des occurrences sont significativement liées à l'âge, c'est-à-dire que les jeunes en pratiquent plus ; ce n'est pas le cas pour la marche assistée. En ce qui concerne les comportements bipèdes non assistés, aucune corrélation n'a été observée avec l'âge. L'unique relation significative trouvée est entre la durée des occurrences de la marche bipède et la masse corporelle et la distribution des masses (Fig. 3a), et ce, même lorsque le contexte d'activité est considéré (c'est-à-dire au cours de l'alimentation, des relations sociales et du jeu). Il semble donc y avoir une relation étroite entre le modèle de distribution des masses et le mécanisme de contrôle de l'équilibre lors de la marche bipède spontanée des babouins (Fig. 3b). L'effet mécanique engendré par le modèle de distribution des masses chez les primates non humains actuels sur leur capacité à réaliser la marche bipède suggère que ce même mécanisme lié à l'inertie de la partie supérieure du corps (c'est-à-dire la partie située au-dessus des hanches) peut avoir été un facteur important dans le contexte de l'évolution des premiers homininés vers la bipédie habituelle. 


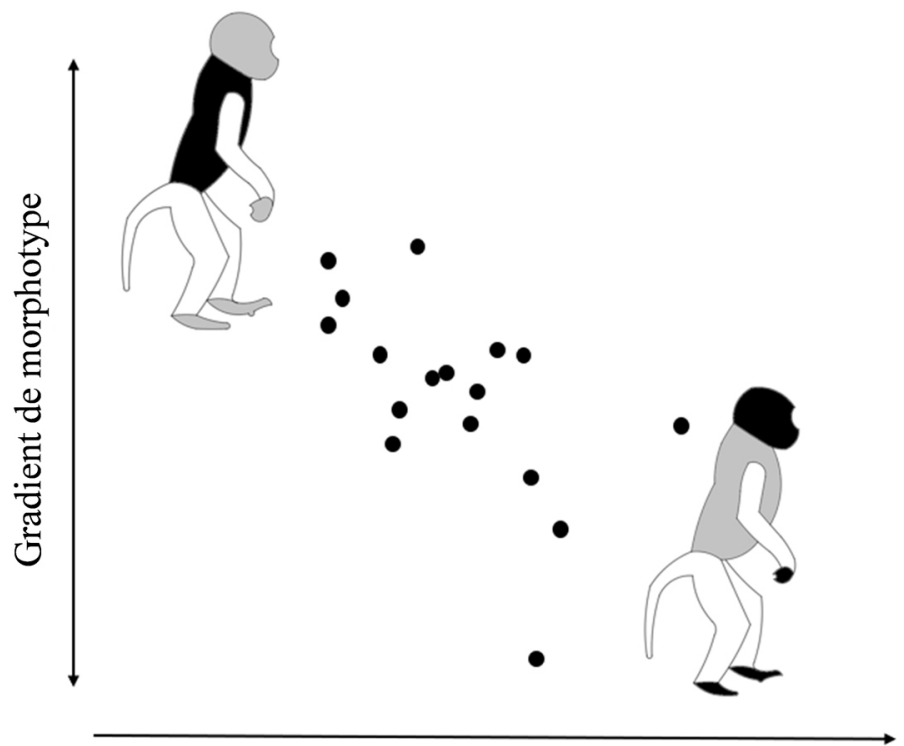

Moyenne individuelle des occurrences bipèdes

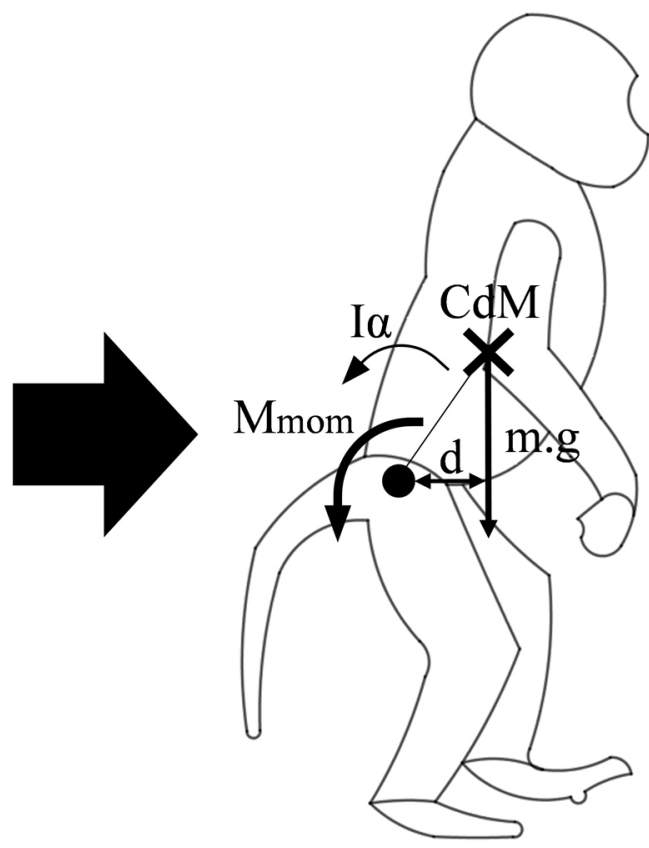

Equation angulaire du mouvement

$$
I \propto=m \cdot g \cdot d+M_{\text {mom }}
$$

Fig. 3 Relation mécanique entre la position du centre de masse et l'équilibre de la marche bipède. Le graphique de gauche montre la relation entre le gradient de variation du morphotype et la moyenne individuelle des occurrences de marche bipède au cours de la croissance du babouin olive. Le modèle de droite représente l'équilibre de la partie supérieure du corps (tête, bras et tronc, c'est-à-dire les parties situées au-dessus de l'articulation de la hanche) dans le plan de progression du mouvement. Cet équilibre est déterminé par l'équation angulaire du mouvement dans laquelle $I=$ le moment d'inertie de la partie supérieure du corps, $\alpha=1$ 'accélération angulaire de la partie supérieure du corps, $m=$ masse de la partie supérieure du corps, $g=-9,81 \mathrm{~m} / \mathrm{s}^{2}$ (accélération gravitationnelle), $d=$ bras de levier de la force gravitationnelle, $M_{m o m}=$ moment de la force musculaire (couple) au niveau de l'articulation de la hanche. CdM est le centre de masse de la partie supérieure du corps / Mechanical relationship between the position of the centre of mass and equilibrium during bipedal walking. The graph on the left shows the relationship between the gradient of morphological variation and the individual mean duration of unassisted bipedal walking in an ontogenetic sample of olive baboons. The model on the right represents HAT (Head + Arm + Trunk) equilibrium in the plane of progression when standing bipedally. This equilibrium is determined by the angular equation of motion: $I=$ the moment of inertia of the HAT, $\alpha=$ the angular acceleration of the HAT; $m=$ mass of the HAT; $g=-9.81 \mathrm{~m} / \mathrm{s}^{2}$ (gravitational acceleration); $d=$ moment arm of the gravitational force, $M_{\text {mom }}=$ muscle torque at the hip. CdM is the centre of mass of the HAT

\section{Morphologie, coordination et contrôle moteur}

\section{Cas de la quadrupédie}

En général, au cours des premiers stades du développement, les éléments temporels de la marche quadrupède changent et sont susceptibles d'être liés aux propriétés morphologiques (taille et forme) et dynamiques (inertie) intrinsèques des membres et du corps. En effet, au cours de l'ontogenèse des espèces nidicoles (lorsqu'un petit est dépendant de soins parentaux avant d'atteindre l'autonomie), bien que la coordination entre les membres soit contrôlée par le système neurologique (générateurs centraux de modèles de mouvement et/ou contrôle supraspinal), les paramètres mécaniques des membres et du corps peuvent avoir un impact sur l'optimisation locomotrice au cours de la marche. Chez le babouin, la convergence des périodes pendulaires naturelles entre les membres antérieurs et postérieurs semble indiquer, d'un point de vue biomécanique, une adaptation à la locomotion quadrupède $[5,23]$. Par ailleurs, il y a une amélioration significative de la coordination en marche quadrupède lors des premiers stades locomoteurs. Cette amélioration, bien que liée à la maturation du système nerveux, semble être initialement facilitée par la convergence de ce paramètre intrinsèque des membres. En d'autres termes, la convergence des périodes pendulaires naturelles des membres a une influence significative et positive sur la mise en place de la 
coordination chez le babouin olive. Cela facilite probablement le contrôle moteur et l'apprentissage de la marche quadrupède. Ensuite, le contrôle neuromoteur semble devenir graduellement important relativement à la dynamique morphologique des membres durant la période infantile. La spécialisation locomotrice du babouin pour la quadrupédie semble donc être présente très tôt, dès les stades précoces de son développement. Dans le contexte socioécologique $\mathrm{du}$ babouin olive, les jeunes doivent être capables de se déplacer très tôt par eux-mêmes puisque, à un an, ils sont sevrés et doivent être capables de suivre de manière autonome la troupe de babouins dans la savane [24].

\section{Cas de la bipédie}

Parallèlement au développement de la quadrupédie et de la coordination des membres, il se produit également un développement de la capacité à utiliser occasionnellement la bipédie chez le babouin. En effet, la coordination lors de la marche bipède augmente avec le développement de la coordination en quadrupédie. L'acquisition de la marche quadrupède n'est donc pas exclusive du développement d'autres modes, ici la marche bipède, et ce, en dépit des expériences bipèdes qui restent rares pendant l'ontogenèse du babouin $(<0,5 \%$ de son répertoire). Ces observations suggèrent un mécanisme de contrôle basique et similaire en bipédie et en quadrupédie chez les primates ; une observation directe de l'activité musculaire au cours de ces différents types de marche est aujourd'hui nécessaire afin de confirmer ce mécanisme. Néanmoins, ce processus, qui semble également être observé chez le chimpanzé [25], questionne dans une perspective évolutive les modalités d'apparition de nouveaux modes locomoteurs au cours de l'évolution des primates, dont l'homme.

\section{Perspective évolutive : l'émergence de la bipédie}

Chez les primates non humains, les modes locomoteurs secondaires, comme la bipédie, dont les jeunes font rapidement l'expérience comme des sous-produits du développement locomoteur, peuvent potentiellement mener à des nouveautés évolutives lorsqu'ils sont sous des pressions de sélection appropriées. Cette information, replacée dans un contexte paléoanthropologique, peut éclairer certains aspects de l'évolution de la bipédie et particulièrement la transition de la bipédie occasionnelle vers une bipédie plus habituelle. En effet, cela suggère que dans le continuum de mouvements observés chez les primates non humains, les modes locomoteurs ne sont pas exclusifs les uns des autres, au contraire. Chez un ancêtre homininé possédant une prédisposition anatomique (par exemple au niveau du morphotype comme une distribution des masses augmentant l'inertie de la partie supérieure du corps, voir ci-dessus), une bipédie plus habituelle aurait pu émerger grâce à ce mécanisme, et ce, dans un répertoire possédant toujours une part non négligeable de quadrupédie. Dans le modèle évolutif proposé par Rose [2] pour le processus de «bipédalisation » des homininés, c'est la diversité des répertoires qui évolue. Différents modes locomoteurs, réalisés dans des proportions importantes, peuvent donc se retrouver au sein du même répertoire positionnel. Par ailleurs, au-delà de la spécialisation à la quadrupédie chez le babouin olive, il existe des compromis morphologiques permettant la réalisation d'un répertoire varié. Plus largement, il semble que le schéma corporel global des primates non humains actuels leur permette de pratiquer la bipédie sans que leur morphologie osseuse n'en présente les marques, tout comme l'homme moderne pratique le grimper vertical, sans que sa morphologie osseuse n'en présente les marques [26,27], mais également la suspension, voire la marche quadrupède pour se déplacer dans des milieux particuliers tels que des pierriers par exemple. Dans ce contexte, on peut supposer que chez un homininé tel que l'Australopithecus afarensis, en présence de plusieurs marques suggérant la bipédie [28,29], la composante bipède devait représenter une proportion significative de son répertoire posturo-locomoteur, tout comme le grimper devait également être un mode important pour fourrager dans les arbres. L'objectif des futurs travaux sur ces questions est d'élargir ces recherches à d'autres modèles de primates non humains afin de proposer, à terme, des proportions d'utilisation de ces différents modes posturo-locomoteurs et ainsi de modéliser les répertoires des premiers homininés.

Remerciements Je souhaite remercier dans un premier temps mes deux encadrants de doctorat, le Pr Peter Aerts et le Dr Gilles Berillon. Je remercie également tous les acteurs de la station de primatologie du CNRS qui m'ont permis de réaliser ce travail dans des conditions optimales. Je remercie les Drs François Marchal et Aurélien Mounier, secrétaire général de la Société d'anthropologie de Paris et membre du comité de rédaction des Bulletins et Mémoires de la Société d'anthropologie de Paris, pour leur relecture attentive de la première version de ce manuscrit ainsi que pour leurs corrections et commentaires qui ont permis de l'améliorer. Enfin, je remercie la Société d'anthropologie de Paris pour son soutien.

Liens d'intérêts : l'auteur déclare ne pas avoir de liens d'intérêts.

\section{Références}

1. Prost JH (1965) A definitional system for the classification of primate locomotion. Am Anthropol 67:1198-214 
2. Rose M (1991) The process of bipedalization in hominids. In: Coppens Y, Senut B (eds) Origine(s) de la bipédie chez les hominidés. Éditions du Centre national de la recherche scientifique, Paris, pp 37-48

3. Hurov JR (1991) Rethinking primate locomotion: what can we learn from development? J Motor Behav 23:211-8

4. Druelle F, Berillon G (2014) Bipedalism in non-human primates: a comparative review of behavioural and experimental explorations on catarrhines. BMSAP 26:1-10

5. Druelle F, Aerts P, D'Août K, et al (2017) Segmental morphometrics of the olive baboon (Papio anubis): a longitudinal study from birth to adulthood. J Anat 230:805-19

6. Witte H, Preuschoft H, Recknagel S (1991) Human body proportions explained on the basis of biomechanical principles. Z Morphol Anthropol 78:407-23

7. Hooper SL (2012) Body size and the neural control of movement. Curr Biol 22:R318-R22

8. Druelle F, Aerts P, Berillon G (2016) Effect of body mass distribution on the ontogeny of positional behaviors in non-human primates: longitudinal follow-up of infant captive olive baboons (Papio anubis). Am J Primatol 78:1201-21

9. Druelle F, Berillon G, Aerts P (2017) Intrinsic limb morphodynamics and the early development of interlimb coordination of walking in a quadrupedal primate. J Zool 301:235-47

10. Druelle F, Aerts P, Berillon G (2016) Bipedality from locomotor autonomy to adulthood in captive olive baboon (Papio anubis): cross-sectional follow-up and first insight into the impact of body mass distribution. Am J Phys Anthropol 159:73-84

11. Leigh SR, VandeBerg JL, Williams-Blangero S, et al (2009) Growth and development of baboons. In: Tardif SD, WilliamsBlangero S (eds) The baboon in biomedical research. Springer, New York, pp 57-88

12. Raichlen DA (2005) Ontogeny of limb mass distribution in infant baboons (Papio cynocephalus). J Hum Evol 49:452-67

13. Myers MJ, Steudel K (1997) Morphological conservation of limb natural pendular period in the domestic dog (Canis familiaris): implications for locomotor energetics. J Morphol 234:183-96

14. Druelle F, Berillon G (2013) Bipedal behaviour in olive baboons: infants versus adults in a captive environment. Folia Primatol $84: 347-61$

15. Kilbourne BM, Hoffman LC (2013) Scale effects between body size and limb design in quadrupedal mammals. PloS One 8:e78392
16. Aerts P, Van Damme R, Van Elsacker L, et al (2000) Spatiotemporal gait characteristics of the hind-limb cycles during voluntary bipedal and quadrupedal walking in bonobos (Pan paniscus). Am J Phys Anthropol 111:503-17

17. Demes B (2011) Three-dimensional kinematics of capuchin monkey bipedalism. Am J Phys Anthropol 145:147-55

18. Kimura T (1996) Centre of gravity of the body during the ontogeny of chimpanzee bipedal walking. Folia Primatol 66:126-36

19. Nakatsukasa M, Hirasaki E, Ogihara N (2006) Energy expenditure of bipedal walking is higher than that of quadrupedal walking in Japanese macaques. Am J Phys Anthropol 131:33-37

20. Pontzer H, Raichlen DA, Rodman PS (2014) Bipedal and quadrupedal locomotion in chimpanzees. J Hum Evol 66:64-82

21. Thompson NE, Demes B, O'Neill MC, et al (2015) Surprising trunk rotational capabilities in chimpanzees and implications for bipedal walking proficiency in early hominins. Nat Commun 6:8416

22. Videan EN, McGrew WC (2002) Body weight and bipedality in captive chimpanzees (Pan troglodytes). Laboratory Primate Newsletter 41

23. Raichlen DA (2004) Convergence of forelimb and hindlimb natural pendular period in baboons (Papio cynocephalus) and its implication for the evolution of primate quadrupedalism. J Hum Evol 46:719-38

24. Rose M (1977) Positional behaviour of olive baboons (Papio anubis) and its relationship to maintenance and social activities. Primates 18:59-116

25. Kimura T, Yaguramaki N (2009) Development of bipedal walking in humans and chimpanzees: a comparative study. Folia Primatol 80:45-62

26. DeSilva J (2009) Functional morphology of the ankle and the likelihood of climbing in early hominins. Proc Natl Acad Sci USA 106:6567-72

27. Venkataraman VV, Kraft TS, Dominy NJ (2013) Tree climbing and human evolution. Proc Natl Acad Sci USA 110:1237-42

28. Stern JT, Susman RL (1983) The locomotor anatomy of Australopithecus afarensis. Am J Phys Anthropol 60:279-317

29. Ward CV (2013) Postural and locomotor adaptations of Australopithecus species. The Paleobiology of Australopithecus. Springer, pp 235-45 